\section{Vaisseaux humains reconstitués par génie tissulaire}

Jean-Claude Stoclet, Karina Laflamme, François A. Auger, Lucie Germain
$>$ Les progrès du génie tissulaire permettent maintenant de reconstituer des vaisseaux sanguins fonctionnels à partir de cellules humaines. Dans des conditions bien précises, ces vaisseaux possèdent une structure, des propriétés mécaniques et des propriétés fonctionnelles (notamment en termes de vasomotricité) qui permettent de les utiliser comme modèles pour contourner les difficultés d'obtention et d'utilisation de vaisseaux humains pour la recherche expérimentale. En effet, l'utilisation de vaisseaux humains à des fins expérimentales est limitée par des problèmes éthiques et par les difficultés d'interprétation des résultats liées à leur hétérogénéité. C'est pourquoi une grande partie des recherches en biologie et en pharmacologie vasculaires est réalisée soit sur des modèles animaux, soit sur des cellules en culture, qui ne sont pas toujours représentatifs des vaisseaux humains. Le génie tissulaire peut apporter une source alternative de vaisseaux humains pour pallier ces inconvénients. «
La finalité du génie tissulaire est de reconstituer des tissus et des organes en laboratoire. Les progrès réalisés dans ce domaine au cours des dernières décennies permettent aujourd'hui d'envisager la production d'organes complexes, tels que les vaisseaux sanguins, à partir de cellules humaines. Initialement conçus dans un but d'autogreffe [1], ces vaisseaux peuvent procurer d'intéressants modèles d'études humains.

\section{Obtention de vaisseaux reconstitués par génie tissulaire}

Différentes méthodes ont été développées afin de reconstituer des vaisseaux dont les caractéristiques histologiques et fonctionnelles se rapprochent de celles des tissus natifs. La première méthode proposée par $C$. Weinberg et $\varepsilon$. Bell [2] est fondée sur l'inclusion de fibroblastes et de cellules musculaires lisses bovines dans des gels de collagène tubulaires, à l'intérieur desquels sont ensemencées

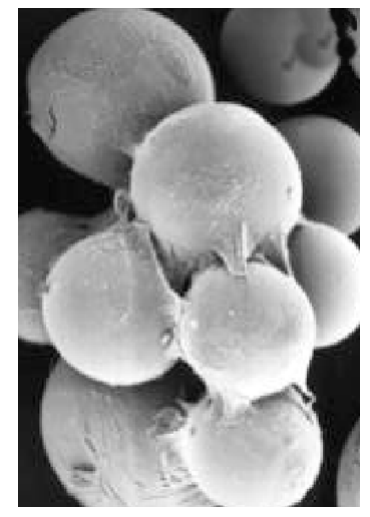

des cellules endothéliales. Des équivalents vasculaires humains ont également été produits par cette méthode [3].

J.C. Stoclet: Laboratoire de pharmacologie et physicochimie des interactions cellulaires et moléculaires, UMR CNRS 7034, Faculté de pharmacie, BP 24, 74, route du Rhin, 67401 IIIkirch, France.

K. Laflamme, F.A. Auger,

L. Germain: Laboratoire d'organogenèse expérimentale (LOEX), département de chirurgie de l'université Laval, Hôpital du Saint-Sacrement du CHA, 1050, chemin Sainte-Foy, Québec, G1S 4L8, Canada.

Lucie.germain@chg.ulaval.ca

Les trois tuniques vasculaires sont reconstituées et présentent un aspect macroscopique et histologique semblable à celui des vaisseaux natifs. Cependant, leur résistance mécanique à l'éclatement est trop faible pour les utiliser dans des études fonctionnelles en pharmacologie ou pour la transplantation. Plusieurs équipes s'efforcent d'améliorer leurs propriétés mécaniques $[4,5]$.

Plus récemment, une autre méthode a été adaptée pour la production de vaisseaux sanguins. II s'agit d'ensemencer des cellules musculaires lisses bovines dans un support biosynthétique tubulaire ajouré fabriqué à partir d'acide polyglycolique. Les cellules remplissent les treillis en se multipliant et en produisant de la matrice extracellulaire. Des cellules endothéliales sont ensuite ensemencées à l'intérieur $[6,7]$. Ces prothèses comprennent seulement deux des trois tuniques vasculaires et présentent les désavantages associés à la présence de biomatériau: il est en effet difficile de dissocier les propriétés des tissus vivants reconstitués de celles du support inerte auquel ils sont intégrés, les biomatériaux pouvant alors interférer dans les mesures.

Notre laboratoire d'organogenèse expérimentale, dont l'objectif est de reconstituer des organes par génie tis- 
sulaire, a développé une approche complètement nouvelle pour produire des vaisseaux reconstitués à partir de cellules seulement, par auto-assemblage (Figure 1). La première étape consiste à obtenir des cultures de chacun des trois types cellulaires, c'est-à-dire de fibroblastes, de cellules musculaires lisses et de cellules endothéliales [3, 8]. Il est essentiel, pour la qualité du tissu final obtenu, que ces cellules soient bien caractérisées à ce stade $[3,8,9]$. Chaque tunique peut être reconstruite et soit analysée séparément, soit incluse dans un vaisseau entier, selon les besoins des applications expérimentales.

\section{Structure et fonction des vaisseaux reconstitués}

\section{Histologie et propriétés mécaniques}

Le vaisseau humain reconstitué par la méthode d'auto-assemblage contient les trois tuniques vasculaires (adventice, média et intima), selon une structure histologique semblable à celle des vaisseaux natifs. La densité du collagène y est particulièrement élevée pour un vaisseau reconstitué. D'autres composantes de la matrice extracellulaire sont également présentes, dont l'élastine dans l'adventice. Un des grands avantages de la reconstitution par auto-assemblage est d'obtenir, en l'absence de tout matériau synthétique, une résistance à l'éclatement suffisante pour permettre la greffe du vaisseau [8] et même le montage en bain d'organe pour étudier sa contraction [10]. D'autres vaisseaux obtenus à partir de cellules animales ont également pu être montés de la même façon, mais leur résistance mécanique à l'étirement était renforcée par un matériau synthétique $[6,7]$ ou hétérologue [11].

\section{Caractères phénotypiques}

des cellules vasculaires

Malgré des différences phénotypiques importantes d'un lit vasculaire à l'autre, les cellules endothéliales et les cellules musculaires lisses vasculaires présentent dans l'organisme un phénotype dont certains caractères sont retrouvés dans tous les vaisseaux normaux. Pour ce qui est des cellules endothéliales, les marqueurs les plus classiques que sont le facteur von Willebrand et l'aptitude à incorporer la lipoprotéine de faible densité acétylée ont été caractérisés dans les cellules endothéliales des vaisseaux reconstitués [8, 11]. En outre, dans ces vaisseaux reconstitués, les cellules endothéliales répondent à des signaux appropriés par une libération de monoxyde d'azote (NO) ou de prostacycline, événement qui se traduit par une inhibition de l'adhésion des plaquettes [8] et une vasodilatation dépendant de l'endothélium [11].
Dans les vaisseaux natifs, les cellules musculaires lisses sont attachées à la matrice extracellulaire, essentiellement composée de fibres de collagène et d'élastine, avec lesquelles elles constituent la tunique média. Elles s'y divisent très peu et existent principalement sous un phénotype contractile, caractérisé par l'expression de protéines contractiles (comme l' $\alpha$-actine musculaire lisse et deux isoformes musculaires des chaînes lourdes de la myosine, SM-1 et SM-2 [12]) et de protéines du cytosquelette (comme la desmine) [13]. Lorsque les cellules sont détachées de leur matrice, par exemple lors de leur mise en culture ou dans des vaisseaux pathologiques, elles acquièrent un phénotype prolifératif sous lequel elles peuvent non seulement proliférer, mais encore sécréter la matrice extracellulaire [14]. Le passage du phénotype contractile au phénotype prolifératif s'accompagne de la perte de l'expression de protéines contractiles et du cytosquelette, ainsi que de protéines impliquées dans la transmission de signaux, tels le canal calcique de type $L[15$ ] sensible aux dihydropyridines et la protéine kinase $G$ responsable des effets du GMP cyclique. Par la suite, la présence de desmine et du système GMP cyclique-protéine kinase $G$ fonctionnel indique que les cellules musculaires lisses, après avoir proliféré et sécrété une matrice, ont retrouvé dans la média reconstituée un phénotype contractile, une fois attachées à cette matrice et enchassées dans celle-ci $[8,10]$.

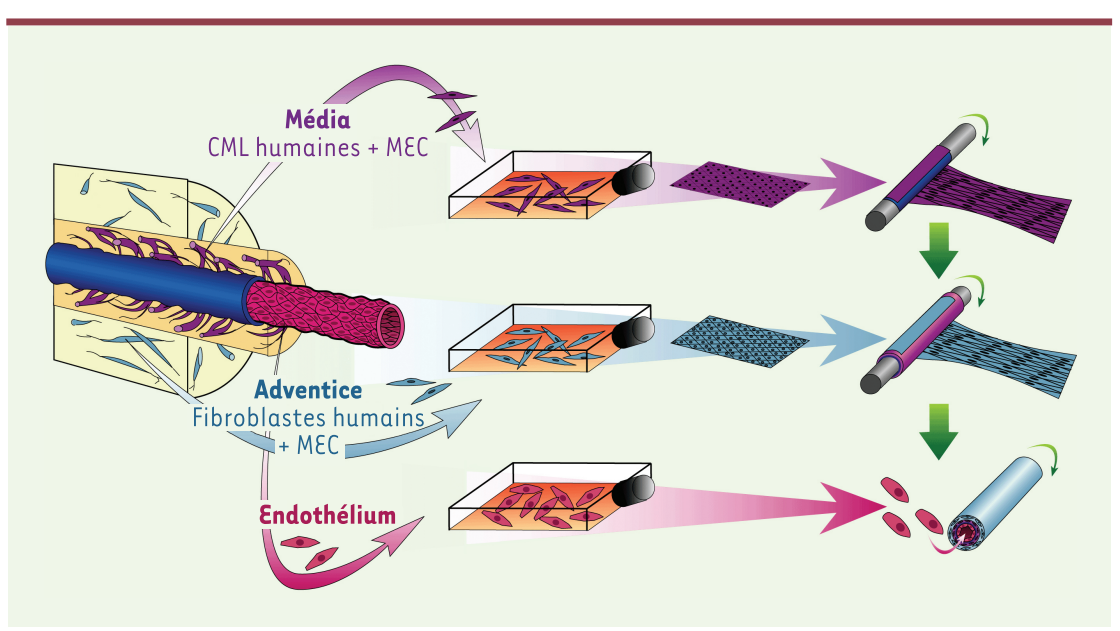

Figure 1. Production de vaisseaux humains par auto-assemblage. Chacun des trois types cellulaires est cultivé à partir de tissus humains; les cellules endothéliales et musculaires lisses (CML) sont respectivement isolées de l'endothélium et de la média du cordon ombilical, et les fibroblastes du derme. Des feuillets tissulaires produits à partir des cellules musculaires lisses (cultivées dans un milieu contenant du sérum et de l'acide ascorbique pour favoriser la formation de la matrice extracellulaire, MEC) et des fibroblastes sont enroulés autour d'une matrice déposée sur un mandrin pour reconstituer la média et l'adventice. Le mandrin est enlevé et les cellules endothéliales sont ensemencées à l'intérieur. Ainsi, les différentes couches du vaisseau sont reconstituées (remerciements à Danielle Larouche pour l'illustration). 


\section{Vasomotricité}

Un aspect essentiel pour l'utilisation de vaisseaux reconstitués en pharmacologie est de connaître les mécanismes de la vasomotricité (vasoconstriction et

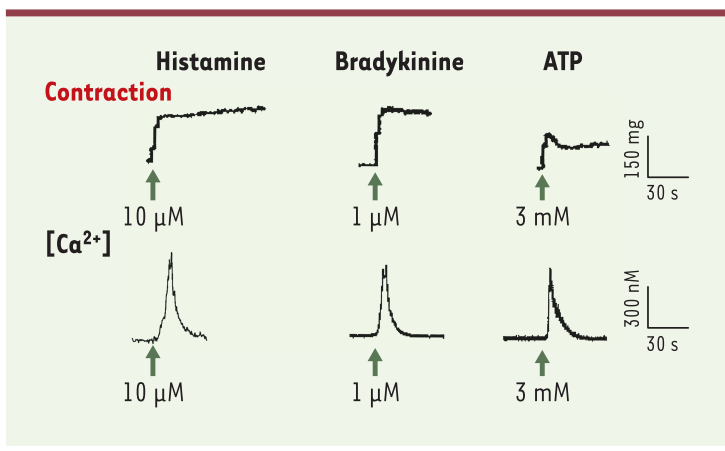

Figure 2. Caractérisation du rôle du calcium dans la vasoconstriction. Les courbes montrent des tracés représentatifs de l'augmentation de la tension (en haut) et de la concentration du calcium (en bas) produite par des agonistes vasonconstricteurs dans la média humaine reconstituée in vitro (d'après [10]). vasodilatation) exprimés dans leurs cellules musculaires lisses. Dans la média humaine reconstituée, les agonistes produisent une augmentation transitoire de la concentration en ions $\mathrm{Ca}^{2+}$ et une contraction soutenue après retour de la concentration en $\mathrm{Ca}^{2+}$ à son niveau de base (Figure 2). II semble que la contraction y soit déclenchée par le $\mathrm{Ca}^{2+}$ et que son maintien fasse appel à un ou plusieurs mécanismes de sensibilisation tels qu'ils ont été décrits dans les vaisseaux natifs. Dans la média humaine reconstituée [10] comme dans les vaisseaux natifs, les mécanismes qui sous-tendent l'élévation de la concentration en $\mathrm{Ca}^{2+}$ comportent un influx de $\mathrm{Ca}^{2+}$ à travers la membrane plasmique et une libération de $\mathrm{Ca}^{2+}$ à partir de réserves intracellulaires. À la différence cependant de ce qui est généralement observé dans les vaisseaux natifs, l'influx de $\mathrm{Ca}^{2+}$ dans la média reconstituée est insensible aux dihydropyridines, suggérant que les cellules musculaires lisses n'y possèdent pas de canaux calciques de type $L$, ou que ceux-ci ne sont pas fonctionnels. Par ailleurs, les cellules musculaires lisses de la média humaine reconstituée sont capables de se relâcher sous l'effet de substances qui augmentent soit l'AMP, soit le GMP cycliques: ainsi possèdent-elles les mécanismes leur permettant de répondre aux facteurs endothéliaux essentiels que sont la prostacycline, qui active l'adénylyl cyclase, et le NO, qui active la guanylyl cyclase.

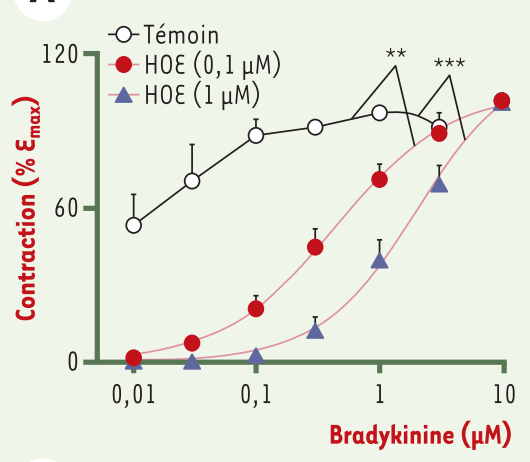

C

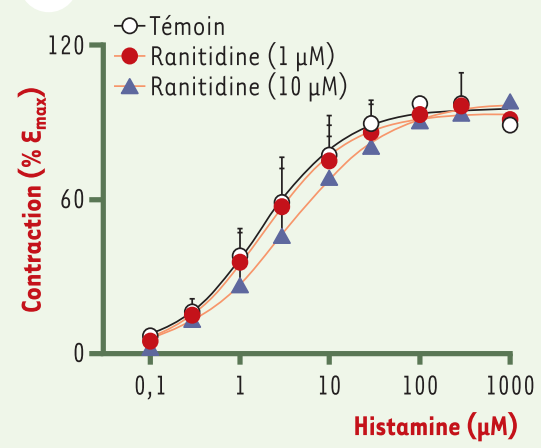

B

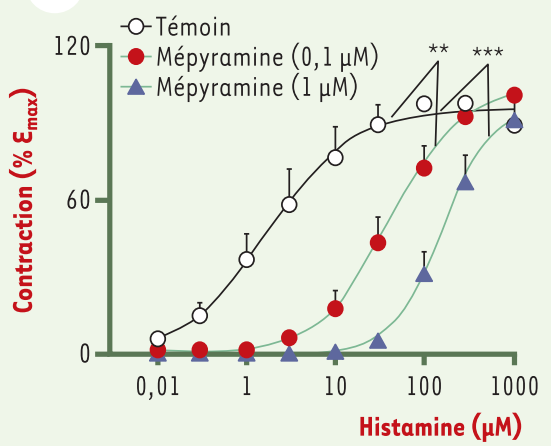

D

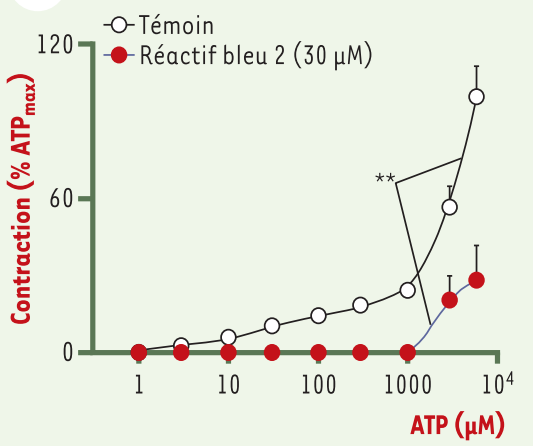

Figure 3. Caractérisation pharmacologique de récepteurs aux agonistes vasoconstricteurs dans la média humaine reconstituée. Les courbes reliant l'effet des agonistes vasoconstricteurs (contraction) à la concentration en agonistes ont été obtenues en l'absence (témoins) et en présence d'inhibiteurs sélectifs. $\boldsymbol{A}$. HOE140, antagoniste des récepteurs $B_{2}$ à la bradykinine; $\boldsymbol{B}$. mépyramine, antagoniste des récepteurs $H_{1}$ à l'histamine; $C$. ranitidine, antagoniste des récepteurs $\mathrm{H}_{2}$ à l'histamine; $\boldsymbol{D}$. réactif bleu, antagoniste des récepteurs purinergiques $P_{2 y 1}$. ${ }^{*} \leq 0,01$, $\star \star \star \leq 0,001$, moyennes \pm de 4 à 6 expériences (d'après [10]).
L'expression de récepteurs est l'un des caractères phénotypiques différant suivant le lit vasculaire. La caractérisation pharmacologique, à l'aide d'agonistes et d'antagonistes appropriés, des récepteurs de la média humaine reconstituée à partir de cellules musculaires lisses de veine ombilicale [10] a permis de montrer que ce vaisseau possède des récepteurs fonctionnels identiques à ceux des cellules musculaires lisses de la veine d'origine (par exemple les récepteurs $B_{2}$ à la bradykinine et les récepteurs $H_{1}$ à I'histamine), mais aussi des récepteurs dont le sous-type est différent (récepteurs vasoconstricteurs à I'ATP appartenant à la famille $P_{2 y}$ dans la média reconstituée, alors qu'ils sont de type $P_{2 x}$ dans la veine native [16]) (Figure 3).

Ainsi, la caractérisation pharmacologique des récepteurs et l'étude des voies de signalisation sous-jacentes conduisent toutes deux à la même conclusion: les cellules musculaires lisses de la média reconstituée par auto-assemblage possèdent de nombreux caractères phénotypiques propres au phénotype contractile des cellules musculaires lisses et particu- 
liers au vaisseau d'origine, mais aussi des caractères différents. L'étude doit donc en être faite au cas par cas.

\section{Conclusions et perspectives}

Par comparaison avec les vaisseaux isolés humains natifs, les vaisseaux obtenus par génie tissulaire permettent de pallier certains inconvénients comme la limitation de leur disponibilité et la variabilité des résultats. Ils possèdent ainsi certains des avantages des cultures cellulaires: peu de problèmes éthiques, obtention de grandes quantités de tissus d'une même origine et meilleure reproductibilité des expériences. Celles-ci peuvent en effet être reproduites autant de fois que nécessaire sur des vaisseaux reconstitués disponibles en quantité suffisante et obtenus à partir de cellules provenant d'un même sujet. Inversement, les vaisseaux natifs, obtenus en quantité souvent limitée (surtout s'ils sont de faible calibre), ne permettent qu'un petit nombre d'expériences sur le même tissu. Les expériences doivent donc être répétées sur des vaisseaux qui, provenant de sujets différents, peuvent être d'une grande hétérogénéité, en raison non seulement de différences génétiques, mais aussi de facteurs parfois incontrôlés propres à chaque patient comme le mode de vie, le régime alimentaire ou encore les traitements médicamenteux. II peut en résulter une importante dispersion des résultats individuels qui rend l'interprétation difficile. Bien entendu, il est également possible, dans un but de comparaison, de reconstituer des vaisseaux à partir de cellules de sujets différents, toutes choses étant identiques par ailleurs (ce qui met à l'abri des variations individuelles d'origine environnementale).

Par comparaison avec des cellules humaines en culture, les vaisseaux reconstitués par auto-assemblage permettent d'obtenir des modèles plus proches du tissu d'origine, avec des cellules plus différenciées possédant une fonction essentielle comme la vasomotricité, et dont l'étude expérimentale est beaucoup plus facile.

Les vaisseaux reconstitués à partir de cellules de veines ombilicales humaines ont plusieurs avantages: facilité relative d'obtention du vaisseau d'origine, techniques bien établies de culture des cellules et d'obtention du vaisseau reconstitué, possibilité d'obtenir une assez grande quantité de matériel à partir d'une seule veine d'origine, connaissances sur le phénotype des cellules dans le vaisseau reconstitué, origine humaine. Cependant, on peut concevoir aussi d'utiliser d'autres cellules vasculaires comme des fragments sains d'artères ou de veines obtenus au cours d'opérations chirurgicales. On peut encore envisager d'employer des cellules progénitrices endothéliales circulantes, comme cela a été récemment réalisé avec succès pour obtenir un vaisseau reconstitué de mouton [11]. Naturellement, le modèle devrait être perfectionné dans l'avenir, par exemple en soumettant son endothélium à un cisaillement approprié, en perfusant le vaisseau pendant sa préparation. On sait en effet que le cisaillement appliqué pendant plusieurs jours induit l'expression de divers gènes [17] et favorise non seulement l'adhérence des cellules endothéliales [11], mais encore l'expression dans celles-ci de molécules importantes comme la NO-synthase [18]. En outre, d'autres types de cellules comme les monocytes/macrophages, par exemple, devraient pouvoir être introduits dans les vaisseaux reconstitués [19]. $\diamond$

\section{SUMMARY}

Human tissue engineered blood vessels: a novel support

for vascular biology and pharmacology research

Progress in tissue engineering now allows the recreation of functional blood vessels from cultured human vascular cells. When reconstructed under specific conditions, their structure, mechanical properties and function (especially vasomotricity) allow them to be used as human models for studying the biology and pharmacology of blood vessels. These models may help to circumvent the limitations in the obtention and use of native human blood vessels for experimental purpose. $\diamond$

\section{RÉFÉRENCES}

1. Auger FA. Le génie tissulaire: du rêve à la réalité. Med Sci (Paris) 2000; 16: 1624-31.

2. Weinberg CB, Bell $\varepsilon$. A blood vessel model constructed from collagen and cultured vascular cells. Science 1986; 231: 397-400.

3. L'Heureux N, Germain L, Labbe R, Auger FA. In vitro construction of a human blood vessel from cultured vascular cells: A morphologic study. J Vasc Surg 1993; 17: 499-509.

4. Seliktar D, Black RA, Vito RP, Nerem RM. Dynamic mechanical conditioning of collagen-gel blood vessel constructs induces remodeling in vitro. Ann Biomed Eng $2000 ; 28: 351-62$.

5. Neidert MR, Lee ES, Oegema TR, Tranquillo RT. Enhanced fibrin remodeling in vitro with TGF-betal, insulin and plasmin for improved tissue-equivalents. Biomaterials 2002; $23: 3717-31$.

6. Niklason LE, Abbott W, Gao J, et al. Morphologic and mechanical characteristics of engineered bovine arteries. J Vasc Surg 2001; 33: 628-38.

7. Niklason $L \varepsilon$, Gao J, Abbott WM, et al. Functional arteries grown in vitro. Science 1999; 284: 489-93.

8. L'Heureux N, Paquet $S$, Labbe $R$, et al. A completely biological tissue-engineered human blood vessel. FASEB J 1998; 12: 47-56.

9. Germain L, Carrier P, Auger FA, et al. Can we produce a human corneal equivalent by tissue engineering? Prog Retin Eye Res 2000; $5: 497-527$.

10. L'Heureux N, Stoclet JC, Auger FA, et al. A human tissue-engineered vascular media: A new model for pharmacological studies of contractile responses. FASEB J 2001; 15: $515-24$.

11. Kaushal S, Amiel GE, Guleserian KJ, et al. Functional small-diameter neovessels created using endothelial progenitor cells expanded ex vivo. Nat Med 2001; 9: $1035-40$.

12. Owens GK. Regulation of differentiation of vascular smooth muscle cells. Physiol Rev 1995; 75: 487-517.

13. Absher M, Woodcock-Mitchell J, Mitchell J, et al. Characterization of vascular smooth muscle cell phenotype in long-term culture. In Vitro Cell Dev Biol 1989; 25: $183-92$.

14. Thyberg J. Differentiated properties and proliferation of arterial smooth muscle cells in culture. Int Rev Cytol 1996; 169: 183-265.

15. Richard S, Neveu D, Carnac G, et al. Differential expression of voltage-gated $\mathrm{Ca}(2+)$-currents in cultivated aortic myocytes. Biochim Biophys Acta 1992; 1160: $95-104$.

16. Bo X, Sexton A, Xiang Z, et al. Pharmacological and histochemical evidence for P2X receptors in human umbilical vessels. Eur J Pharmacol 1998; 353: 59-65.

17. Davies PF, Barbee KA, Volin MV, et al. Spatial relationships in early signaling events of flow-mediated endothelial mechanotransduction. Annu Rev Physiol 1997; $59: 527-49$

18. Ranjan V, Xiao Z, Diamond SL. Constitutive NOS expression in cultured endothelial cells is elevated by fluid shear stress. Am J Physiol 1995; 269: 550-5.

19. Petitclerc $\varepsilon$, Levesque L, Grose JH, et al. Pathologic leukocyte infiltration of the rabbit aorta confers a vasomotor effect to chemotactic peptides through cyclooxygenase-derived metabolites.

TIRÉS À PART Immunol 1996; 156: 3426-34. 